\title{
Craniofacial dysplasia-osteopenia syndrome
}

INSERM

\section{Source}

INSERM. (1999). Orphanet: an online rare disease and orphan drug data base.

Craniofacial dysplasia-osteopenia syndrome. ORPHA:314555

Craniofacial dysplasia-osteopenia syndrome is a rare, genetic developmental defect during embryogenesis disorder characterized by craniofacial dysmorphism (incl. brachycephaly, prominent forehead, sparse lateral eyebrows, severe hypertelorism, upslanting palpebral fissures, epicanthal folds, protruding ears, broad nasal bridge, pointed nasal tip, flat philtrum, anteverted nostrils, large mouth, thin upper vermilion border, highly arched palate and mild micrognathia) associated with osteopenia leading to repeated long bone fractures, severe myopia, mild to moderate sensorineural or mixed hearing loss, enamel hypoplasia, sloping shoulders and mild intellectual disability. 